\title{
Cytoplasmic Immunoglobulin Content in Multiple Myeloma
}

\author{
Bart Barlogie, Raymond Alexanian, Mark Pershouse, Leslie Smallwood, and Lon Smith \\ The University of Texas System Cancer Center, M. D. Anderson Hospital and Tumor Institute, \\ Texas Medical Center, Houston, Texas 77030
}

\begin{abstract}
Bone marrow cells of 82 patients with multiple myeloma were subjected to flow cytometric analysis of DNA and cytoplasmic immunoglobulin (CIg) content using propidium iodide and direct immunofluorescence assays. Except for two patients with nonsecretory myeloma, there was conformity in the immunoglobulin type derived from immunoelectrophoresis and plasma cell Clg staining. One patient with nonsecretory myeloma exhibited monotypic CIg staining, while the second showed no reaction. In eight patients with IgG lambda myeloma, the same tumor cells contained both lambda and kappa light chains, suggesting the productive rearrangement of both light chain genes. 14 patients with previously unrecognized plasma cells of low RNA content, all of whom were resistant to chemotherapy, were identified by $\mathrm{Clg}$ staining. By revealing previously unrecognized plasma cells with low RNA content, CIg analysis identified more patients with treatment-refractory myeloma.
\end{abstract}

\section{Introduction}

The plasma cells of patients with multiple myeloma usually contain an abnormal DNA and an increased RNA content, permitting the quantitation of plasma cells by flow cytometry (1-3). Higher response rates to both initial and salvage therapy have been noted with increasing RNA content $(4,5)$, and a shorter survival time has been found in patients with marked bone marrow plasmacytosis (4). A typical feature of plasma cells is the presence of cytoplasmic immunoglobulin $(\mathrm{CIg}){ }^{1}$ We have developed a flow cytometric assay for the concurrent analysis of DNA and CIg content in order to quantify plasma cells when studies of DNA and RNA content could not provide a clear distinction from normal cells. Whether $\mathrm{Clg}$ staining might clarify the biology of plasma cells in patients with nonsecretory disease or with other atypical clinical features was also evaluated.

\section{Methods}

Studies were performed in 82 patients with advanced stages of multiple myeloma including 23 without prior therapy. The bone marrow of all

Address reprint requests to Dr. Barlogie, Department of Hematology, 6723 Bertner-Box 55, Houston, TX 77030.

Received for publication 28 November 1984 and in revised form 5 March 1985.

1. Abbreviations used in this paper: $\mathrm{Clg}$, cytoplasmic immunoglobulin; $\mathrm{CV}$, coefficient of variation.

J. Clin. Invest.

(c) The American Society for Clinical Investigation, Inc. 0021-9738/85/08/0765/05 $\$ 1.00$

Volume 76, August 1985, 765-769 patients contained at least $7 \%$ plasma cells on routine morphological examination. For flow cytometry studies, bone marrow aspirates were separated by Hypaque-Ficoll gradient centrifugation (6). Interphase cells were collected and washed once in phosphate-buffered saline. One aliquot of cells was always stained with the metachromatic dye acridine orange for concomitant DNA and RNA analysis using a mercury arc flow cytometer (Phywe Co., Gottingen, Germany) (7). Routinely, 10,000 cells were analyzed and tumor cells gated on the basis of abnormal DNA and RNA content $(1,4)$. In this manner, tumor cell DNA and RNA index values were derived from the relation to normal lymphocytes (specifically, the ratio of median fluorescence channel numbers of tumor to normal diploid cells) (1). The proportion of cells with abnormal DNA and/or RNA content in the entire sample was also determined.

A second bone marrow aliquot was processed for biparametric DNA-Clg analysis with an EPICS V flow sorter (Coulter Electronics, Inc., Hialeah, FL). Cells were fixed in $70 \%$ ice-cold ethanol for at least $24 \mathrm{~h}$. Single cell suspensions were then exposed separately to antilight-chain and anti-heavy-chain reagents (fluorescein-conjugated $\mathrm{F}\left(\mathrm{ab}^{\prime}{ }^{\prime}{ }_{2}\right.$ fragments; Capell Laboratories, Westchester, PA) at dilutions of 1:200 $(0.1 \mathrm{mg} / \mathrm{ml})$ and counterstained for DNA with propidium iodide (8). In three instances of dual light chain reaction, different antisera were evaluated in the same manner (Tago Inc., Burlingame, CA).

Successive flow cytometric analyses were performed on 10,000 cells, each stained for both light chains, and, in a subset of patients, stained also for two heavy chains (typically, IgG and IgA). Except for eight instances of dual light chain reaction, marked differences in staining intensity were observed between pairs of samples stained for light and heavy chains (see Fig. 1). In the $\sim 80 \%$ of patients with DNA-abnormal stemlines, bright $\mathrm{Clg}$ fluorescence was usually limited to aneuploid cells, while residual diploid cells showed the same dim fluorescence observed after staining with the opposite light chain reagent. The proportion of tumor cells was readily determined by applying an electronic gating procedure to the brightly stained cells with an abnormal DNA content (Fig. 1). To obtain a measure of $\mathrm{Clg}$ content for each patient, a Clg index was computed from the ratio of median $\mathrm{Clg}$ fluorescence intensities of aneuploid and diploid $G_{1}$ cells of the same sample.

For the remaining DNA-diploid samples, both the percentage of plasma cells and their CIg index were determined in comparison with the nonspecific staining pattern of cells reacted with the opposite light chain antiserum. Specifically, kappa and lambda distribution curves were superimposed electronically to identify the lower level of specific $\mathrm{Clg}$ fluorescence. The $\mathrm{CIg}$ index was then computed from the ratio of median intensities of specific and nonspecific (opposite light chain) fluorescence. The degree of heterogeneity of $\mathrm{CIg}$ content among plasma cells from the same patient was expressed as a coefficient of variation (CV) of the monotypic Clg distribution. The CV (percent) was computed from the ratio of the standard deviation and the mean of $\mathrm{Clg}$ fluorescence channel numbers times 100 .

There were eight patients with dual cytoplasmic light chain expression. In the six aneuploid cases, all DNA-abnormal cells demonstrated both kappa and lambda staining. This finding and fluorescence microscopic evaluation (after double-staining with fluorescein- and rhodamineconjugated antisera) indicated that the same plasma cells reacted with both light chain antisera. As in the majority of cases with monotypic $\mathrm{Clg}$ staining, the $\mathrm{Clg}$ index was computed in relation to dimly stained normal diploid cells. In the two cases of diploid myeloma with dual light chain staining, assessment of the degree of marrow plasmacytosis 
and of the $\mathrm{Clg}$ index was performed in comparison with normal bone marrow samples separately stained with kappa and lambda light chain antisera.

\section{Results}

Fig. 1 shows a typical example of bivariate flow cytometric DNA-CIg analysis of bone marrow cells from a patient with IgG kappa myeloma. A bright staining reaction was observed in hyperdiploid cells using anti-gamma and anti-kappa sera, but not with anti-alpha and anti-lambda reagents. A second example illustrates a patient with concurrent multiple myeloma and acute myelogenous leukemia (Fig. 2). Compared with normal marrow, two discrete DNA stemlines (diploid and hyperdiploid) with markedly increased RNA content were identified. Using light chain antisera, only the hyperdiploid DNA stemline contained monoclonal $\mathrm{CIg}$ of kappa type, and hence, represented the myeloma tumor population; the diploid DNA stemline showed similar weak staining reactions with both kappa and lambda reagents and represented the leukemic clone.

Table I summarizes the relationship between $\mathrm{CIg}$ and immunoelectrophoretic results. Concordance of light chain phenotypes was observed in 80 of 82 patients, including 18 patients on whom additional heavy chain analyses were conducted. Two patients lacked a monoclonal protein by immunoelectrophoresis, but showed marrow involvement microscopically ( 25 and $42 \%$ ) and by DNA-RNA flow cytometry (18 and $69 \%$ ). One patient had kappa staining within the cytoplasm ("low secretor"), while the second showed no detectable anti-light-chain reaction ("low producer"). A third
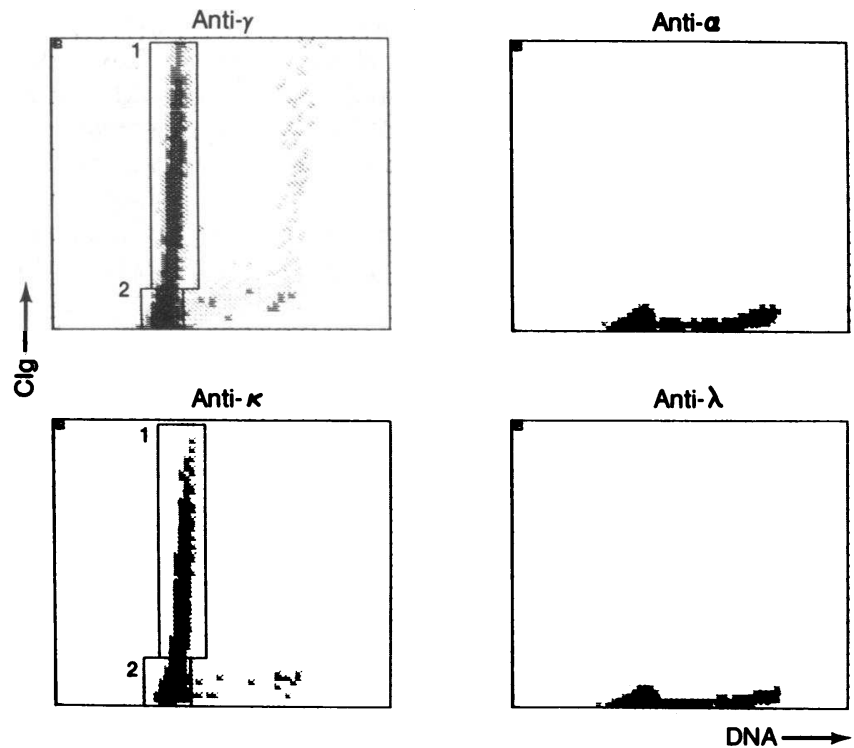

Figure. 1. Myeloma phenotyping by Clg flow cytometry. Bone marrow cells were stained with anti-heavy and anti-light chain reagents and counterstained for DNA with propidium iodide. Note the positive reaction of hyperdiploid $G_{1}$ cells (window 1) only with antigamma and anti-kappa and not with anti-alpha and anti-lambda antisera, confirming the presence of IgG kappa monoclonal protein. The $\mathrm{Clg}$ index as a measure of plasma cell $\mathrm{Clg}$ content was computed in relation to nonspecific fluorescence (window 2).
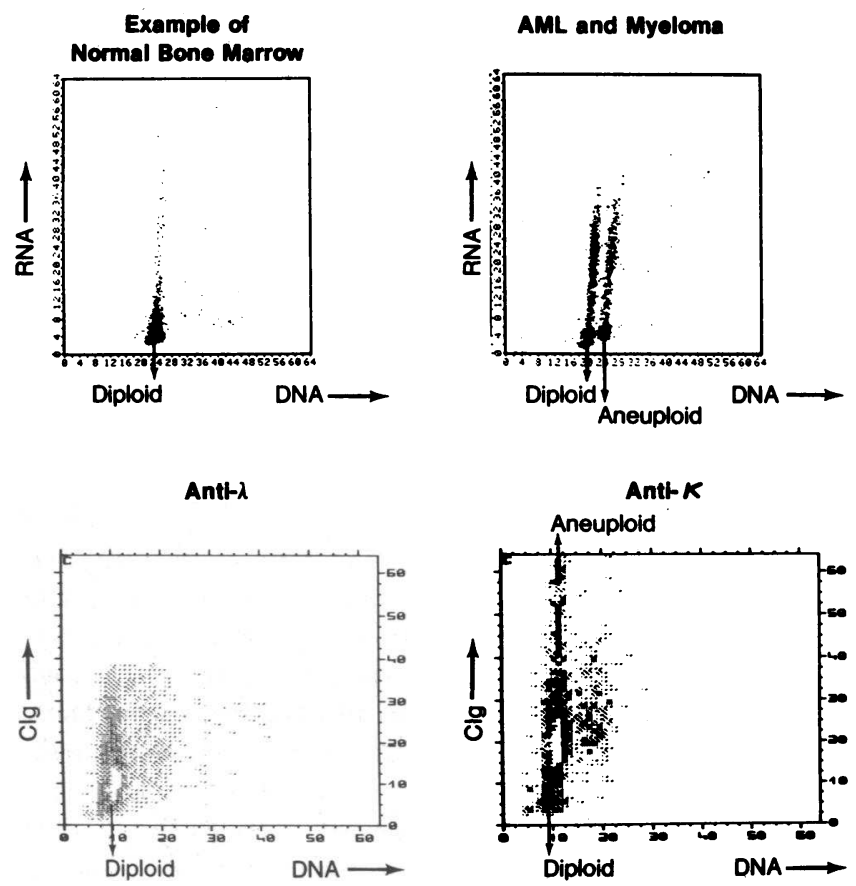

Figure 2. Comparison of DNA-RNA and DNA-Clg analysis in a patient with concurrent myeloma and acute myeloid leukemia (AML). Upper panels represent DNA-RNA histograms; lower panels show DNA-CIg histograms. Compared with normal bone marrow with a prevalence of diploid cells with low RNA content (upper left), both diploid and hyperdiploid DNA stemlines with markedly increased RNA content were found in a patient with both myeloma and AML (upper right). DNA-CIg analyses with anti-lambda and anti-kappa reagents revealed monoclonal kappa expression in the hyperdiploid DNA stemline, whereas diploid cells showed nonspecific staining. Hence, the hyperdiploid clone represented myeloma, and the diploid cells with high RNA content represented AML cells (see reference 7). Compared with the example in Fig. 1, nonspecific fluorescence (lower left) is shifted toward higher channel numbers due to instrument setting. The CIg index of specific kappa fluorescence is relatively low (lower right).

Table I. Immunoglobulin Typing by Immunoelectrophoresis and CIg Analysis using Flow Cytometry

\begin{tabular}{|c|c|c|c|c|}
\hline \multirow{2}{*}{$\begin{array}{l}\text { Clg flow } \\
\text { cytometry }\end{array}$} & \multicolumn{4}{|c|}{ Immunoelectrophoresis } \\
\hline & Kappa & & oda & No reaction \\
\hline Kappa & 45 & & & $1^{*}$ \\
\hline Lambda & \multicolumn{4}{|c|}{35} \\
\hline No reaction & & & & $1 \ddagger$ \\
\hline Heavy chain§ & G & $\mathbf{A}$ & D & No reaction \\
\hline G & 11 & & & $1^{\prime \prime}$ \\
\hline $\mathbf{A}$ & & 5 & & \\
\hline D & & & 1 & \\
\hline
\end{tabular}

* Low secretor.

$¥$ Low producer.

$\S$ Subset of 18 patients in whom heavy chain analyses were also conducted.

"Patient with lambda light chain in cytoplasm and urine; however, gamma heavy chain was only detected in cytoplasm and not in serum. 
patient with a positive reaction of cells for lambda light chain and with Bence Jones proteinuria also exhibited cytoplasmic IgG in the absence of a monoclonal serum peak.

Fig. 3 illustrates the correlations between the proportions of tumor cells identified by DNA-RNA or DNA-CIg flow cytometry and those enumerated by morphology for the 77 patients in whom all three assays were available. Both cytometric assays showed similar degrees of plasmacytosis in the 48 patients with DNA aneuploidy, where abnormal cells were clearly separated from normal cells $(\mathrm{R}=0.83, \quad P$ $<0.001$ ) (Fig. $3 A$ ). Among the 29 patients with diploid myeloma, there were 8 patients with a DNA-RNA pattern indistinguishable from normal marrow but a monoclonal light chain reaction was evident in $5-69 \%$ of cells (median, $26 \%$ ). These cells corresponded to plasma cells on microscopic examination (Fig. $3 \mathrm{~B}$ ). One patient with nonsecretory myeloma showed 60-70\% plasma cells with high RNA content but lacked a monotypic CIg pattern ("low-producing myeloma"). There were also 6 patients with aneuploid myeloma, in whom a second diploid DNA stemline with $5-20 \%$ plasma cells was uncovered by monotypic CIg staining (four kappa and two lambda) (Fig. 4). Thus, the combined analysis of DNA-CIg and DNA-RNA permitted the identification of previously unrecognized cell populations with low RNA content in $\sim 20 \%$ of our patients.

Eight patients with a discrete DNA-RNA abnormality had plasma cells which expressed both kappa and lambda light chains. Immunoelectrophoresis revealed monoclonal IgG lambda in all instances. An example of such double-staining is illustrated in Fig. 5 with three different DNA stemlines in the diploid, the low degree hyperdiploid, and the tetraploid range, all of which expressed kappa, lambda, and IgG immunoglobulin (the latter not shown). Fluorescence microscopy revealed concurrent kappa and lambda reactions in the same plasma cells. The dual light chain reaction was confirmed in three patients using a different source of anti-light-chain antisera.

There was marked variation in the CIg staining per plasma cell in individual patients, which was expressed as a $\mathrm{CV}$ ranging from 30 to $80 \%$ (median, 50\%). To compare the CIg content per cell among different patients, a $\mathrm{CIg}$ index was defined from the ratio of the median fluorescence intensities of specific vs. nonspecific anti-light-chain reactions. Among 23 patients studied at diagnosis, the $\mathrm{CIg}$ index ranged from 2 to 27 , with a median value of 10 . There was no correlation between the RNA and the CIg index, but the $\mathrm{CIg}$ index decreased with increasing marrow tumor infiltrate $(R=-0.25$, $P<0.01)$.

We also examined the clinical implications of $\mathrm{CIg}$ analysis. Among the 23 previously untreated patients, neither $\mathrm{CIg}$ index nor $\mathrm{CIg}$ dispersion (CV) correlated with response to chemotherapy. By CIg analysis, we had identified 14 patients with a low RNA index (less than four times the RNA content of normal lymphocytes) in diploid plasma cells, either as their sole tumor cell population (8 patients) or in addition to an aneuploid DNA stemline (6 patients). Five individuals in each category were evaluated after 1-3 mo of resistance to initial chemotherapy (primary unresponsive disease); the remaining patients were studied at diagnosis, and none responded to standard chemotherapy. Thus, $\mathrm{CIg}$ analysis unmasked diploid plasma cells with low RNA content, which was associated with complete resistance to preceeding or subsequent chemotherapy.
A

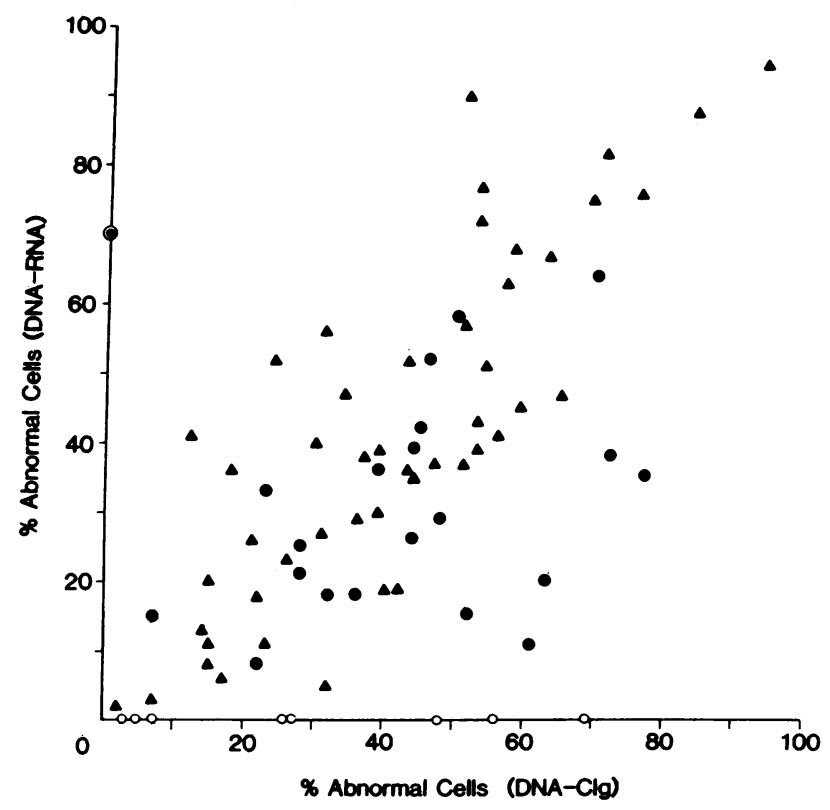

Figure 3. Correlations between the proportion of tumor cells in the bone marrow identified by flow cytometry of DNA-RNA and DNA$\mathrm{Clg}$ as well as by microscopy. (A) Close concordance between DNARNA and DNA-Clg cytometric assays in aneuploid myeloma ( $\mathbf{\Delta}$ ). Among the 29 diploid cases $(\bullet, 0)$, there were 8 with undetectable plasma cells on DNA-RNA histograms $(A)$ but with monotypic $\mathrm{Clg}$ staining (O), consistent with monoclonal plasma cells $(B) . \bullet, \circ, \odot$;

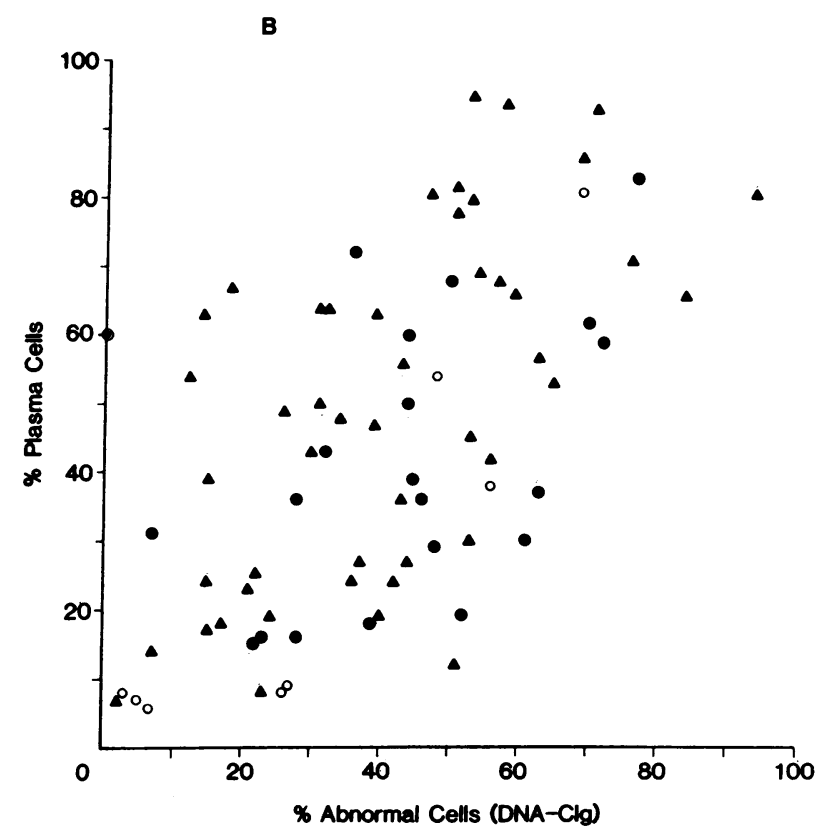

Diploid, $n=29, r=0.40, P<0.01$. $\triangle$; Aneuploid, $n=48, r=0.83$, $P<0.01$. (B) Correlation between marrow plasmacytosis by microscopy and by DNA-CIg cytometry. One patient lacked a monotypic Clg reaction, but had a high RNA content with typical plasma cell

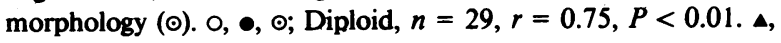
Aneuploid, $n=48, r=0.62, P<0.01$. 


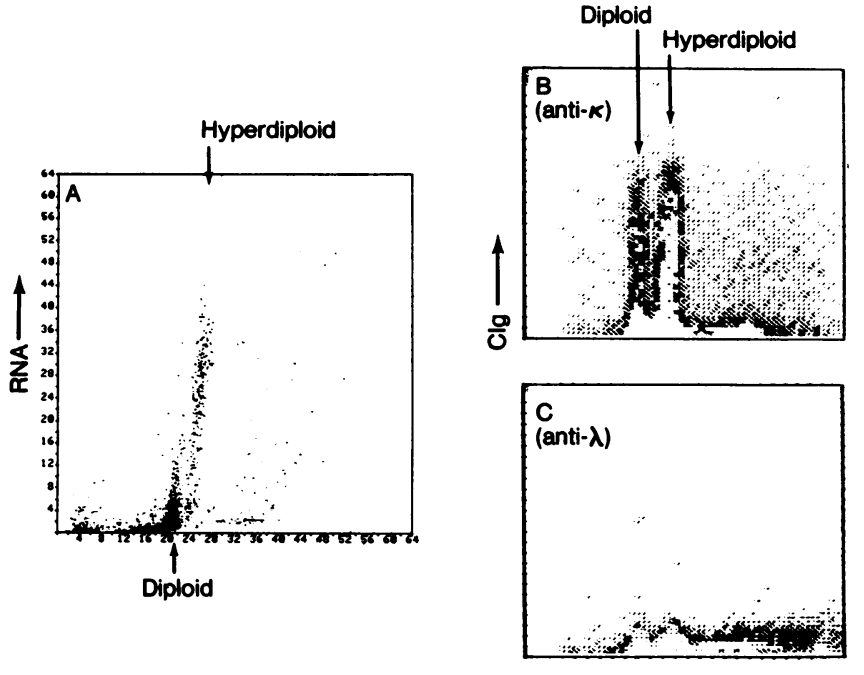

DNA $\longrightarrow$

Figure 4. Biclonal myeloma DNA stemlines identified by DNA-CIg flow cytometry. $(A)$ DNA-RNA analysis demonstrates only one cell population with increased RNA and DNA content, as typically found in myeloma (4). (B) Monoclonal light chain reaction with anti-kappa serum in both diploid and hyperdiploid DNA stemlines. $(C)$ Nonspecific staining with anti-lambda serum.

\section{Discussion}

Plasma cells represent the last phase of B lymphocyte differentiation and are readily identified by the presence of monotypic immunoglobulin in the cytoplasm $(\mathrm{Clg})$ and, more recently, by monoclonal antibodies reacting specifically with the surface membrane of plasma cells $(9,10)$. While flow cytometry has been used to probe the lymphocyte differentiation pathway, this method has not been applied to assess the biologic and clinical relevance of $\mathrm{Clg}$ in patients with multiple myeloma (11). Using a direct immunofluorescence technique, there was excellent agreement between immunoelectrophoresis and Clg flow cytometry in immunoglobulin phenotyping. In conjunction with morphology and DNA-RNA flow cytometry, DNA-Clg analysis helped define special categories of "low-secretory" and "low-producing" myeloma.

There were eight instances of concurrent kappa and lambda reaction in the same aneuploid cells. This observation must be interpreted with caution, as all eight patients produced lambda light chains. The dual staining reaction, however, was confirmed in three patients using a different reagent; in one of those examined further, we only eliminated the lambda, but not the kappa staining of the doubly stained cells by preincubation with free lambda light chains. Thus, our findings seem consistent with those rare reports of coexpression of kappa

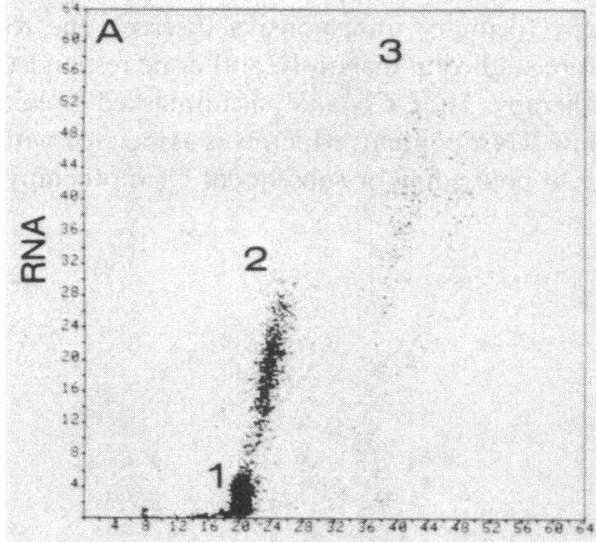

DNA

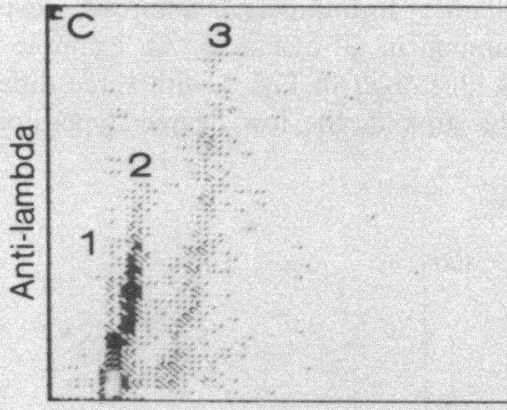

DNA

DNA

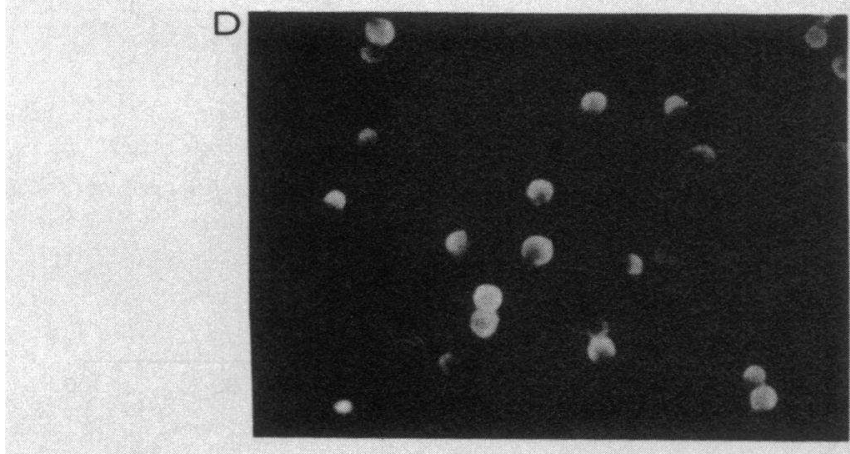

FITC

Figure 5. Dual light chain expression in myeloma. (A) DNA-RNA analysis reveals three discrete populations with diploid (1), low degree hyperdiploid (2), and near tetraploid DNA stemlines (3), with typically increased RNA content in populations (2) and (3). (B), $(C)$ DNA-CIg analysis demonstrates positive reactions in all three DNA

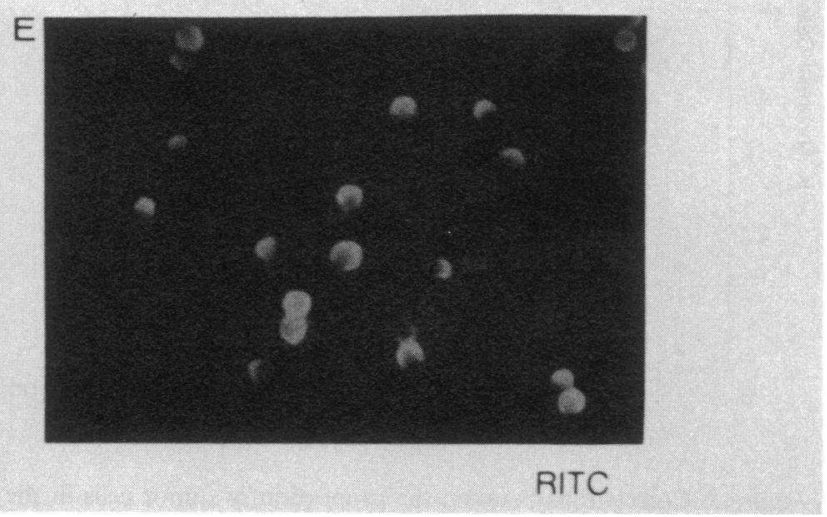

stemlines with both kappa $(B)$ and lambda $(C)$ reagents. $(D),(E)$ On microscopic inspection of doubly stained cells (kappa-fluorescein isothiocyanate, $D$; lambda-rhodamine isothiocyanate; $E$ ), the same plasma cells react with both light chain reagents. 
and lambda light chains by the same tumor cells (12-14). Interestingly the dual light chain expression was only observed in lambda-secreting myeloma. This fits the concept of a hierarchy of light chain activation, where kappa gene rearrangement precedes that of lambda $(15,16)$. Among $18 \mathrm{~B}$ cell samples examined from patients with chronic lymphocytic leukemia and long term Epstein-Barr virus-transformed normal human B cell lines, lambda constant region genes remained in the germ line configuration in all eight samples producing kappa light chains (16). In contrast, 10 lymphocyte samples producing lambda light chains showed deletion of both kappa constant region alleles in 9 instances and productive rearrangement of 1 kappa allele in 1 B cell line. The latter case indicates the possibility of productive rearrangement of both light chain genes in lambda-secreting cells. Our Clg flow cytometric data suggest that this event may occur in as many as $10 \%$ of patients with myeloma.

There was close conformity in the degree of bone marrow involvement by tumor cells using morphologic and flow cytometric assays. The latter have the advantage of objectivity and reproducibility. DNA-CIg analysis identified some patients with apparently normal bone marrow on DNA-RNA studies, whose plasma cells were now recognized with a very low RNA content. This assay also revealed diploid plasma cells with a low RNA content in addition to aneuploid cells previously demonstrated by DNA-RNA analysis. Both of these observations are of major importance because of the established resistance to chemotherapy of myeloma patients either with a low RNA index or multiple DNA stemlines $(4,5,17)$.

As we had noted with RNA content, the CIg content per plasma cell varied markedly within and among patients. In previously untreated patients, increasing bone marrow plasmacytosis was associated with a decrease in $\mathrm{CIg}$ index and RNA index (18), which might reflect less differentiated and perhaps more aggressive features of myeloma. The resistance to chemotherapy of low RNA index myeloma has been established $(4,5,17)$ and may be related in part to a greater degree of RNA heterogeneity with increasing tumor burden $(18,19)$. Similar inferences cannot yet be made for $\mathrm{CIg}$ index and $\mathrm{CIg}$ dispersion in view of the small number of patients evaluated at diagnosis.

In summary, DNA-CIg flow cytometry aids in several aspects of myeloma research and clinical management. We were able to recognize patients with "low-secretory" and "lowproducing" disease. The biological significance of $\mathrm{Clg}$ dispersion and the coexpression by the same tumor cell of both light chains requires further study. The importance of objective and quantitative assessment of the degree of marrow tumor infiltration has been demonstrated $(4,17)$. DNA-CIg analysis is superior to DNA-RNA cytometry for assessing marrow plasmacytosis because monoclonal $\mathrm{CIg}$ is more specific for abnormal plasma cells. In conjunction with DNA-RNA flow cytometry, $\mathrm{CIg}$ analysis permitted the identification of more patients with a poor prognosis, whose plasma cells either had a low RNA content, biclonal DNA abnormalities, or both.

\section{Acknowledgments}

This work was supported by National Cancer Institute grants CA 16672, CA28771, and CA37161.

\section{References}

1. Barlogie, B., J. Latreille, D. Swartzendruber, L. Smallwood, A. Maddox, A. M. Raber, B. Drewinko, and R. Alexanian. 1982. Quantitative cytology in myeloma research. In Clinics in Haematology. W. R. Schmidt, editor. W. B. Saunders Co. 19-45.

2. Bunn, P., S. Krasnow, R. Makuch, M. Schlam, and G. Schechter. 1982. Flow cytometric analysis of DNA content of bone marrow cells in patients with plasma cell myeloma. Clinical implications. Blood. 59:528-535.

3. Barlogie, B., M. Raber, J. Schumann, T. Johnson, B. Drewinko, D. Swartzendruber, W. Gohde, M. Andreeff, and E. Freireich. 1983. Perspectives in cancer research: flow cytometry in clinical cancer research. Cancer Res. 43:3982-3997.

4. Barlogie, B., R. Alexanian, E. Gehan, L. Smallwood, T. Smith, and B. Drewinko. 1983. Marrow cytometry and prognosis in myeloma. J. Clin. Invest. 72:853-861.

5. Barlogie, B., L. Smith, and R. Alexanian. 1984. Effective treatment of advanced multiple myeloma refractory to alkylating agents. N. Engl. J. Med. 310:1353-1356.

6. Boyum, A. 1968. Isolation of mononuclear cells and granulocytes from human blood. Scand. J. Clin. Lab. Invest. 21:77-89.

7. Barlogie, B., J. Latreille, E. J. Freireich, C. T. Fu, D. Mellard, M. Meistrich, and M. Andreeff. 1980. Characterization of hematologic malignancies. Blood Cells. 6:719-744.

8. Crissman, H. A., P. F. Mullaney, and J. A. Steinkamp. 1975. Methods and applications of flow systems for analysis and sorting of mammalian cells. Methods Cell Biol. 9:179-246.

9. Anderson, K. C., E. K. Park, M. P. Bates, R. C. F. Leonard, R. Hardy, S. F. Schlossman, and L. M. Nadler. 1983. Antigens on human plasma cells identified by monoclonal antibodies. J. Immunol. 130: 1132-1136.

10. Ruiz-Arguelles, G. J., J. A. Katzmann, P. R. Greipp, N. J. Gonchoroff, J. P. Garton, and R. A. Kyle. 1984. Multiple myeloma: circulating lymphocytes that express plasma cell antigens. Blood. 64: 352-356.

11. Zeile, G. 1980. Intracytoplasmic immunofluorescence in multiple myeloma. Cytometry. 1:37-41

12. Bouvet, J. P., D. Buffe, R. Oriol, and P. Liacopoulos. 1974. Two myeloma globulins, IgG1- $\kappa$ and $\operatorname{IgG} 1-\alpha$, from a single patient (Im). Immunology. 27:1095-1101.

13. Hopper, J. E. 1977. Comparative studies on monotypic IgM and IgG from an individual patient. IV. Immunofluorescent evidence for a common clonal synthesis. Blood. 50:203-211.

14. Choi, Y., and M. Wong. 1981. Double light chain production by leukemic cells of common clonal origin: a case report with a review of pertinent literature. Am. J. Hematol. 11:93-98.

15. Korsmeyer, S. J., P. A. Hieter, J. V. Ravetch, D. G. Poplack, T. A. Waldmann, and P. Leder. 1981. Developmental hierarchy of immunoglobulin gene rearrangements in human leukemic pre-B-cells. Proc. Natl. Acad. Sci. USA. 78:7096-7100.

16. Hieter, P. A., S. J. Korsmeyer, T. A. Waldmann, and P. Leder. 1981. Human immunoglobulin kappa light-chain genes are deleted or rearranged in lambda-producing B cells. Nature (Lond.). 290:368-372.

17. Barlogie, B., R. Alexanian, L. Smith, D. Dixon, L. Smallwood, and K. Delasalle. 1985. Prognostic implications of tumor cell DNA and RNA content in multiple myeloma. Blood. In press.

18. Barlogie, B., and R. Alexanian. Cellular aspects of myeloma: biologic and clinical implications. In Multiple Myeloma and Other Paraproteinemias. I. W. Delamore, editor. Churchill Livingstone Publishers. In press.

19. Goldie, J., and A. Coldman. 1979. A mathematical model for relating the drug sensitivities of tumors to their spontaneous mutation rate. Cancer Treat Rep. 63:1717-1733. 